\title{
Cardiovascular, lactate and appetite response to light and spicy music tempo after an endurance swimming protocol in young girls
}

Javad Mehrabani $^{1 \mathrm{ABCDE}}$, Soodabeh Bagherzadeh ${ }^{2 \mathrm{BCDE}}$, Aboozar Jorbonian ${ }^{1 \mathrm{CD}}$, Eisa Khaleghi-Mamaghani ${ }^{1 \mathrm{DE}}$, Maryam Taghdiri $^{3 \mathrm{D}}$, Mona Mehdizadeh-Haghighi ${ }^{\mathrm{B}}$

${ }^{1}$ University of Guilan, Rasht, Guilan, Iran

${ }^{2}$ Islamic Azad University, Rasht, Guilan, Iran

${ }^{3}$ University of Mazandaran, Babolsar, Mazandaran, Iran

Authors' Contribution: A - Study design; B - Data collection; C - Statistical analysis; D - Manuscript Preparation; E - Resource Collection.

\section{Abstract}

Purpose:

Results:

Conclusions:

Keywords:
During exercise, the effects of music on the performance have been previously evaluated. However, the superiority of the type of music and during recovery is not yet clear. Therefore the aim of this study was to determine the impact of music with a spicy and light beat on changes in lactate levels, blood pressure, heart rate, and appetite during the recovery period after the endurance swimming.

Thirteen healthy young girls participate in three control and experimental trials. The participants performed a swimming. Immediately after swimming, they listened to music. Also, evaluations before and after (several times) swimming were performed.

Five minutes after swimming there was a significant difference between the non-sound group with the music groups $(p<0.05)$. In blood lactate, there was a significant difference between the spicy and light music groups compared to the non-sound group, 2 and 5 minutes after swimming. In heart rate, systolic blood pressure and appetite components, there were significant difference between spicy and light music groups at time 10,15 and 25 minutes. In the 25 minutes after the swim, reducing the heart rate in light music was more than spicy. Also, 10 minutes after swimming, the spicy music group could not cope with the increase in heart rate $(p<0.05)$. There was a significant difference between the two music groups in minutes 5, 10 and 15 after swimming $(p<0.05)$.

Listening to light music during recovery from endurance swimming was associated with decreased lactate levels and heart rate, but listening to spicy music increased heart rate and prospective. swimming, lactate, heart rate, appetite, music tempo.

\section{Introduction}

Athletes use music to improve performance and increase motivation. The effects of listening to music on physiological, psychological and physical indices of sports enthusiasts have also been studied [1-4]. Some studies have shown a positive effect of music on improving the physiological status, such as changes in heart rate (HR), respiration, blood pressure (BP), endorphin levels, skin reactions, brain waves, and also the reduction of sensation and threshold of physical pain [3-5].

The effect of music before and during the sport and physical activities has been discussed before, and It has been shown that music before the exercise can act as a stimulant to the level of arousal and physiological state [6]. Also, the music during exercise has been considered as a motivation to continue long-term, repetitive activities [7].

Endurance exercise and fatigue are associated with changes in the cardio-respiratory system and increase psychological stress [8]. In recent research, submaximal and medium level exercise was improved by music. Also in these exercises music reduced the rating of perceived exertion (RPE) [9, 10].

\footnotetext{
O Javad Mehrabani, Soodabeh Bagherzadeh, Aboozar Jorbonian, Eisa Khaleghi-Mamaghani, Maryam Taghdiri,

Mona Mehdizadeh-Haghighi, 2020

doi:10.15561/26649837.2020.0401
}

In intense exercises, music increases the potential to improving motivation, while this improvement in motivation is likely not to improve performance [11].

Although athletes report music favorable effects during training or competition, conflicting studies have also been observed. The contradictory results of the music effects are probably due to the type of music, the nature of exercise and the specification of participants (age, gender, etc.).

Swimming is a competitive recreational sport that causes significant physiological fatigue from intense activity in competitive swimmer [12]. However, the characteristic of swimming exercise is that the joints are not under bodyweight pressure and its beneficial role in achieving cardiovascular fitness is important. So this sport has been popular with people and athletes and the potential of music is likely effective in swimmer performance. In addition, swimming is a pleasure sport. So it is important to create a feeling of enjoying music in swimming. Yet due to the lack of effective devices for transmitting sound in this environment, the effect of music on swimmer performance is unclear [12].

Appetite is a mental feeling of hunger, fullness, satisfaction and prospective that can affect to calorie intake [13]. Physical activity reduces appetite by increasing energy consumption, and temporarily inhibiting hunger 
and delaying the onset of food eating [14].

Many studies have examined the effects of exercise on appetite, but there is a contradiction in this. Several studies have reported an increase, decrease, and loss of appetite after exercise [13-15]. However, metabolic substances produced by metabolism after an exercise can affect appetite. Studies have shown that increasing lactate due to exercise may be acting a role in reducing appetite and is one of the possible mechanisms for inhibiting hunger [14].

Lactate is a metabolic product of physical activity and it was suggested at the beginning of the twentieth century that increased levels of lactate in muscle and so blood is the major cause of fatigue exertion when exercise is very intense [16].

However, fatigue results in impaired physiological functions. For example, it reduces appetite and accumulates metabolic products. In such a situation, a desirable recovery can lead to the reconstruction of energy resources and delay the fatigue process [7].

Nowadays, the effects of post-exercise music are considered as a contributing factor to recovery. The use of music to improve post-exercise recovery has different physiological dimensions. Using music in the recovery period is considered a new strategy and elite athletes use music before or after the competition to increase their recovery and physiological and psychological fitness [7].

In this regard Jeffreys argued that music was not just a motivator during hard work and could help to better recover. He showed those who listened to music during the recovery period had a faster drop in lactic acid than those who did not listen to music [17].

From a long time ago recovery is performed both active and passive. Active recovery is preferred because it reduces lactate levels in the blood $[18,19]$.

A possible mechanism for this is the increase in blood flow to tired muscles and the metabolites washout by this process. However, most athletes after exercise and the resulting fatigue tend not to perform active recovery with maintaining a certain speed [20]. In this case, using music to facilitate the recovery process seems more appropriate, because it causes a series of changes in emotional, hormonal, nervous and cardiac and cardiorespiratory systems and etc. [7, 20].

Most research in music has focused on the impact of its function on the science, and rarely has been investigated from the biological and psychological point of view [16]. Some limited studies have examined the effect of music on time spent on post-exercise recovery [7]. Also, some researchers have studied the effect of music on the performance of athletes. So, recent studies have mostly focused on the effectiveness of music in reducing stress and enhancing exercise performance [21, 22].

Choosing the right type of music is very important, as the specific type of music stimulates people, and it makes people calm $[23,24]$. So, the main question for researchers is, which music (with what beat) is better.

Therefore, the present study was designed to investigate the effect of the type of music. In this study, we hypothesized that the type of music can have different effects. According to this assumption, we evaluated the effect of music with a spicy and light beat on changes in lactate, BP, HR and appetite during the recovery after an endurance swimming.

\section{Materials and methods}

Participants. Twenty-seven girl swimmers (with 5 years of championship history) voluntarily notification their readiness to participate in the present study. Of these twenty-seven, only twelve (age: $31.25 \pm 3.15 \mathrm{yr}$, height: $165.1 \pm 4.7 \mathrm{~cm}$, weight: $63.1 \pm 3.42 \mathrm{~kg}$, body fat: $26.41 \pm 2.24 \%$, BMI: $22.12 \pm 3.24 \mathrm{~kg} . \mathrm{m}^{2}$ ) were randomly selected to participate in the study. The characteristics of the participants are presented in Table 1.

Prior to the implementation of the research protocol, all stages of the study and possible risks were explained to the participants, and written consent was obtained from them.

The criteria for entering and exiting the participants were as follows:

- Participants were swimmers (with 5 years of championship history).

- Participants were healthy (self-report of their health and health visit by a physician).

- The participants were not smokers and did not drink alcohol. Caffeine or any stimulant was prohibited.

- Participants had no exercise or heavy physical activity 72 hours before taking part in the study.

\section{Initial evaluation}

One week before the start of the protocol, baseline evaluation was performed, which included weight, height, body fat, and waist to hip ratio (WHR). Also, the baseline study was performed to determine a pressure range equal to $75-85 \%$ of the maximum heart rate (MHR) in all participants. In addition the speed of movement along the pool was calculated.

Measurement of appetite

The appetite visual analog scale (VAS) was presented before, immediately after, 15, 30 minutes and 1, 2 and 3 hours after swimming. Participants were asked to fill out a questionnaire at a specified time and on VAS evaluate their amount of fullness and hunger.

Participants on the chart $(0-100 \mathrm{~mm}$ in size $)$ indicated their amount of satisfaction, fullness, hunger, and desire to eat with a marking.

\section{Measurement of heart rate and blood pressure}

Baseline HR and systolic blood pressure (SBP) and diastolic blood pressure (DBP) was measured 75 min after breakfast and before the protocol began. Furthermore, HR and BP was measured immediately after the start of music play and in the 2, 5, 10, 15 and 25 minutes.

\section{Measurement of blood lactate}

Lactate was measured immediately, 5 and 15 minutes after exercise in recovery period using an Xpress lactometer (Germany, Morfelden-Walldorf).

\section{Nutrition control}

Participants ate breakfast similar to 185 calories (fruit juice, bread, and cheese). 


\section{Swimming protocol}

Each participant was present in the pool for 3 sessions. The protocol was repeated with a one-week recovery based on a crossover design for spicy or light music and control (non-sound) trials.

- Each swim session was as follows: 10-min warmup, which included $5 \mathrm{~min}$ of stretching and $5 \mathrm{~min}$ of soft swimming.

- Participants began swimming. Then they continued to until exhaustion with $75-80 \%$ of their MHR.

- Swimming protocol included a high-intensity interval freestyle swimming in a pool with a length of 25 meters. Every 25 meters (pool length) lasted for 55 seconds, and participants every 25 meters had rest for 10 seconds. In the end, participants swam averaged 1240 meters in 24 minutes.

The intensity of exercise was monitored by a HR telemetry device and measured with a Pro chest strap polar HR monitor (H10 polar, 2017, Polar Electro, Inc. Finland).

A 6-20 RPE that introduce by Borg was used to determine the exhaustion of subjects together with HR measures. Borg scale was shown to subjects every 10 seconds (Rest interval time).

Music selection

Music intervention was started immediately after leaving the water. Each music trial included $25 \mathrm{~min}$ light $(80 \mathrm{bpm})$ or spicy $(120 \mathrm{bpm})$ or non-sound (just using headphones without any sound). Each music trial included six unblemished music that played with Adobe Music 1.5 audition software to proportional to the number of rhythms. The music loudness was considered as $70 \mathrm{~dB}$ [7].

It is worth mentioning that the subjects were present in the pool from 8 am (start the research) to $12 \mathrm{pm}$ (end the research) and the pool water temperature was $25-27^{\circ} \mathrm{C}$.

Statistical analysis

The Shapiro-Wilk test was used to determine the normality of data. The results of the study are reported as mean and standard deviation. To determine the variation of each of the variables in different stages was used one-way repeated-measures ANOVA. In addition, the Bonferroni test was used for the difference between test stages and between the groups. The significance level of $\mathrm{P}<0.05$ was considered.

\section{Results}

Blood lactate

Lactate increased immediately after swimming and five minutes after swimming than before swimming in all three groups. Five minutes after swimming there was a significant difference between the no sound group with the spicy and light music group. Also, 5 minutes after swimming there was a significant difference between all three groups with immediately after swimming. In addition, 15 minutes after swimming there was a significant decrease compared to immediately after swimming $(\mathrm{p}<0.05)$ (Table 2).

\section{Heart rate}

In three modes of spicy, light and non-sound music, the HR increased significantly immediately after swimming, $2,5,10,15$ minutes after swimming compared to preswimming. Also, 25 minutes after swimming, the HR significantly decreased in the spicy and light music group than immediately after swimming $(\mathrm{p}<0.05)$ (Table 3$)$.

There were no significant differences between the 5 , 10, and 15 minutes (Table 3).

Two minutes after swimming, there was a significant difference between the spicy and light music groups compared to the non-sound group. This indicates a

Table 1. Characteristics of participants $(n=12)$

\begin{tabular}{ll}
\hline Variable & Mean \pm SD \\
\hline Age $(\mathrm{yr})$ & $31.25 \pm 3.15$ \\
Height $(\mathrm{cm})$ & $165.1 \pm 4.7$ \\
Weight $(\mathrm{kg})$ & $63.1 \pm 3.42$ \\
BMI $\left(\mathrm{kg} \cdot \mathrm{m}^{2}\right)$ & $22.12 \pm 3.24$ \\
Body fat (\%) & $26.41 \pm 2.24$ \\
WHR & $0.79 \pm 0.09$ \\
Swimming distance (m) & $1231.48 \pm 80.12$ \\
Swimming time (min) & $24.02 \pm 3.71$ \\
Note: BMI= Body mass index; WHR= Waist to hip ratio; SD= Standard deviation \\
\hline
\end{tabular}

Table 2. The changes of lactate

\begin{tabular}{llllll}
\hline & Condition & Before & Immediately after & Min 5 & Min 15 \\
\hline \multirow{2}{*}{ Lactate } & Spicy music & $2.19 \pm 0.08$ & ${ }^{*} 3.89 \pm 0.51$ & ${ }^{*}{ }^{+} 4.12 \pm 0.9$ & $3.87 \pm 0.57$ \\
(mmol/L) & Light music & $2.17 \pm 0.04$ & ${ }^{*} 3.45 \pm 0.27$ & ${ }^{*}{ }^{+} 4.01 \pm 1$ & $3.65 \pm 0.15$ \\
& Non-sound & $2.21 \pm 0.06$ & ${ }^{*} 3.66 \pm 0.43$ & ${ }^{*+} 4.89 \pm 0.89$ & ${ }^{+} 4.03 \pm 0.67$ \\
\hline
\end{tabular}

*Significant change with ANOVA Rep. measurements and Bonferroni compared to baseline $(p<0.05)$.

${ }^{+}$Significant change compared with immediately after swimming $(p<0.05)$.

${ }^{\odot}$ Significant difference with non-sound control group 5 min after swimming $(p<0.05)$. 
decrease in HR with both kinds of music in a time of 2 minutes after a swim. Also, there was a significant difference between spicy and light music groups at ten, fifteen and twenty-five minutes. This showed that in all three times, light music was effective in reducing HR $(\mathrm{p}<0.05)$ (Table 3$)$

However, in the twenty-five minutes after the swim, due to a significant reduction in the HR in the light music group compared to the non-sound group, the light music was more effective than spicy. Also, ten minutes after swimming, it was found that because of the significant difference between the spicy music group and the nonsound group, the spicy music group could not cope with the increase in HR $(\mathrm{p}<0.05)$ (Table 3$)$.

Blood pressure

SBP and DBP increased in both spicy and light music groups immediately after swimming and 2 minutes after swimming, in addition, SBP changes significantly in both spicy and non-sound groups 10 min after swimming and non-sound group $15 \mathrm{~min}$ after swimming with before swimming $(\mathrm{p}<0.05)$ (Table 3$)$.

In values heart rate, significant differences were observed between the spicy and light music groups at 5 , 10 and 15 minutes after swimming $(\mathrm{p}<0.05)$ (Table 3$)$.

The values of DBP in all three groups and on times 5th, 10th, 15th and 25th minutes after swimming did not show any significant changes with times before swimming (Table 3).

\section{Appetite}

Fullness

Significant differences were observed between the spicy and light music groups with the pre-swim stage at one and 3 hours after swimming $(\mathrm{p}<0.05)$. Indeed, a significant differences were observed between the spicy and light music groups with an immediately afterswimming stage at fifteen minutes after swimming. In addition, there was a significant difference between the spicy and non-sound music groups at the 15th minute after swimming $(\mathrm{p}<0.05)$. However, at other stages there was no significant difference (Figure $1 \mathrm{~A}$ ).

\section{Hunger}

Significant differences were observed between the spicy and light music groups with the pre-swim stage at 3 hours and 15 min after swimming $(p<0.05)$. Significant differences were observed between the spicy and light music groups with an immediately after-swimming stage at thirty minutes and one, 2 and 3 hours after swimming $(\mathrm{p}<0.05)$. However, at other stages there was no significant difference (Figure $1 \mathrm{~B}$ ).

\section{Prospective}

Significant differences were observed between the spicy and light music groups with an immediately afterswimming stage at fifteen and thirty minutes and one, 2 and 3 hours after swimming. In addition, there was a significant difference between the spicy and light music groups at the 15 th minute $(\mathrm{p}<0.05)$. However, at other stages there was no significant difference (Figure $1 \mathrm{C}$ ).

Satisfaction

Significant differences were observed between the spicy music group with the pre-swim stage at 3 hours after swimming $(\mathrm{p}<0.05)$.

Significant differences were observed between the spicy music group with an immediately after-swimming stage at thirty minutes and 3 hours after swimming. In addition, there was a significant difference between the spicy and light music group with a non-sound group at the 15 th minute after swimming. Also, there was a significant difference between the light and spicy music groups with the non-sound group at 30 minutes and one hour after swimming respectively $(\mathrm{p}<0.05)$. However, at other stages there was no significant difference (Figure $1 \mathrm{D})$.

\section{Discussion}

Previous research has examined the effect of music during and after exercise $[6,25]$. Listening to music during exercise is not possible for some sports including swimming, and swimmers listen to music just before the exercise or during recovery. However, choosing the type of music can also have different effects [23, 24]. According to our knowledge, the effect of music on the recovery period has not been studied.

Two recovery methods have been introduced: this is active and inactive recovery. Many coaches tend to have active recovery because of the superiority of active recovery in reducing lactate $[18,19]$. However, during the recovery period, athletes do not tend to be active recovery and use new methods and techniques to aid in the recovery process [20]. One of these is listening to music.

Table 3. Changes in HR and BP before and after swimming protocol

\begin{tabular}{|c|c|c|c|c|c|c|c|c|}
\hline \multirow{2}{*}{ Variable } & \multirow{2}{*}{$\begin{array}{l}\text { Music } \\
\text { tempo }\end{array}$} & \multirow{2}{*}{$\begin{array}{l}\text { Before } \\
\text { swimming }\end{array}$} & \multirow{2}{*}{$\begin{array}{l}\text { Immediately } \\
\text { after }\end{array}$} & \multicolumn{5}{|l|}{ Recovery } \\
\hline & & & & $\min 2$ & $\min 5$ & $\min 10$ & $\min 15$ & $\min 25$ \\
\hline \multirow{3}{*}{$\mathrm{HR}$ (min) } & Spicy & $96.3 \pm 6.1$ & ${ }^{+} 177.6 \pm 6.8$ & ${ }^{+*} 128.6 \pm 15.3$ & ${ }^{+} 129.8 \pm 9.2$ & $\#+*^{*+} 121.5 \pm 10.8$ & ${ }^{t \#} 112.3 \pm 8.6$ & $\# 96.2 \pm 10.1$ \\
\hline & Light & $95.4 \pm 7.3$ & ${ }^{+} 180.5 \pm 8.2$ & ${ }^{+*} 123.9 \pm 18.1$ & ${ }^{\dagger} 124.7 \pm 11.9$ & ${ }^{\dagger} 116.7 \pm 8.8$ & ${ }^{\dagger} 106.5 \pm 10.4$ & 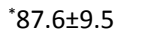 \\
\hline & Non-sound & $93.9 \pm 8.7$ & ${ }^{+} 181.5 \pm 8.2$ & ${ }^{+} 140.3 \pm 14.9$ & ${ }^{+} 121.5 \pm 8.2$ & ${ }^{+} 117.7 \pm 10.4$ & ${ }^{\dagger} 110.8 \pm 12.4$ & $95.6 \pm 8.5$ \\
\hline \multirow{3}{*}{$\begin{array}{l}\text { SBP } \\
(\mathrm{mmHg})\end{array}$} & Spicy & $102.5 \pm 0.7$ & ${ }^{\dagger} 141.4 \pm 1.09$ & ${ }^{\dagger} 130.7 \pm 0.93$ & ${ }^{\#+1} 119.3 \pm 0.96$ & ${ }^{\#+} 120.2 \pm 1.01$ & 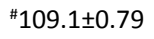 & $106.43 \pm 1.05$ \\
\hline & Light & $100.3 \pm 0.88$ & ${ }^{+} 139.9 \pm 1.19$ & ${ }^{\dagger} 127.9 \pm 0.94$ & ${ }^{+} 114.01 \pm 1.01$ & $111.6 \pm 0.79$ & $102.2 \pm 0.83$ & $104.31 \pm 0.7$ \\
\hline & Non-sound & $101.3 \pm 0.5$ & ${ }^{+} 142.2 \pm 0.2$ & ${ }^{\dagger} 131.5 \pm 1.0$ & ${ }^{\dagger} 118.10 .86$ & ${ }^{\dagger} 122.1 \pm 0.88$ & ${ }^{\dagger} 111.4 \pm 0.91$ & $109.71 \pm 0.9$ \\
\hline \multirow{3}{*}{$\begin{array}{l}\text { DBP } \\
(\mathrm{mmHg})\end{array}$} & Spicy & $7.75 \pm 0.46$ & ${ }^{+} 9.25 \pm 1$ & ${ }^{\dagger} 8.81 \pm 1.28$ & $7.93 \pm 0.62$ & $7.87 \pm 0.58$ & $7.81 \pm 0.75$ & $7.75 \pm 0.65$ \\
\hline & Light & $7.5 \pm 0.75$ & ${ }^{\dagger} 9.12 \pm 1.06$ & ${ }^{+} 8.68 \pm 0.7$ & $7.54 \pm 0.92$ & $7.01 \pm 0.79$ & $7.11 \pm 0.45$ & $7.44 \pm 0.39$ \\
\hline & Non-sound & $7.61 \pm 0.66$ & ${ }^{+} 9.56 \pm 1$ & ${ }^{+} 8.99 \pm 1.28$ & $7.81 \pm 0.12$ & $7.32 \pm 0.58$ & $7.54 \pm 0.48$ & $7.18 \pm 0.09$ \\
\hline
\end{tabular}

Note: HR: Heart rate; SBP: Systolic blood pressure; DBP: Diastolic blood pressure; *significant difference with non-sound group; \#significant difference with light music group. 'Significant change compared with before swimming $(p<0.05)$. 

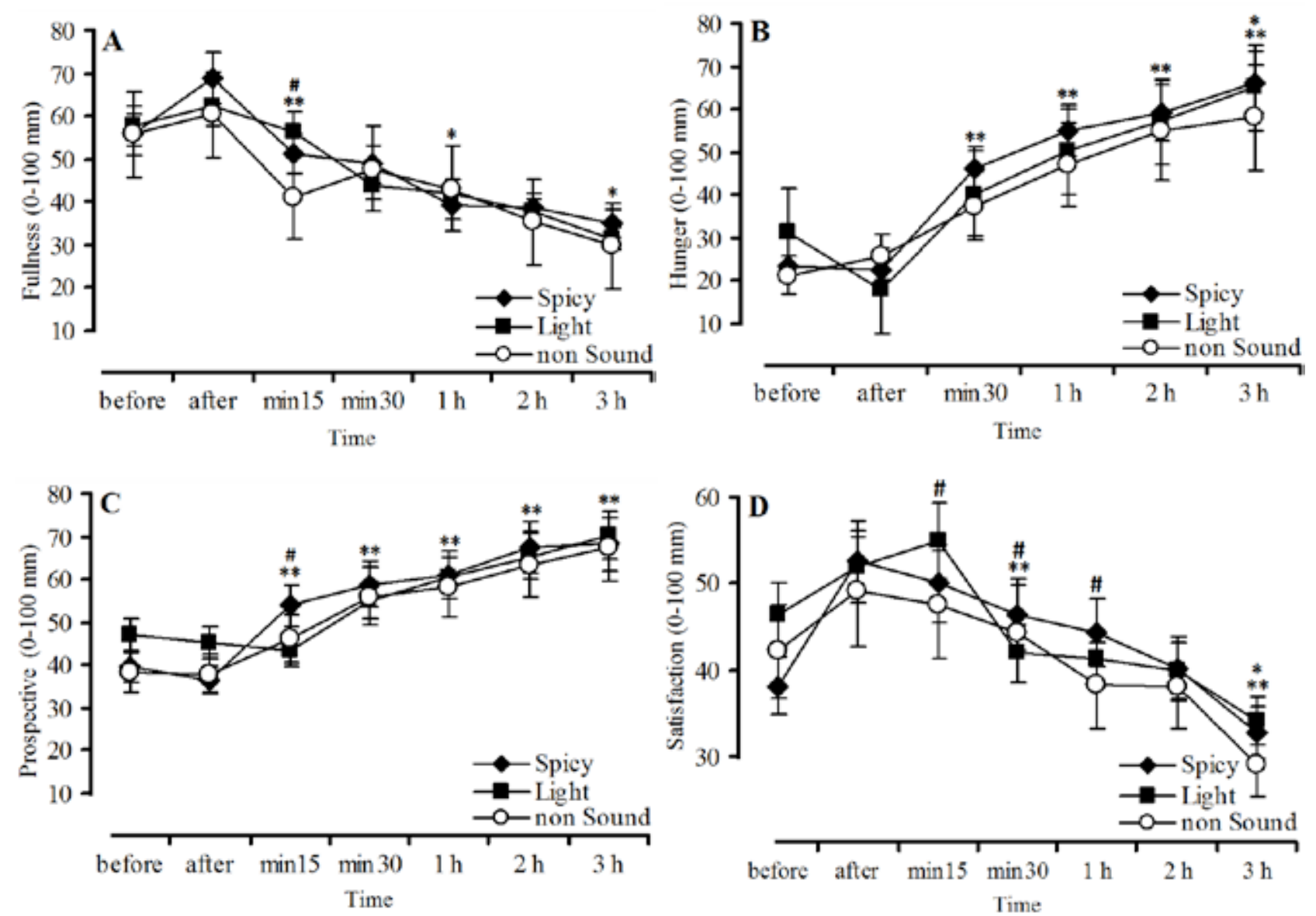

Figure. (A) Fullness; *Significant change in spicy and light groups compared to before and ${ }^{* *}$ immediately after swimming; \#Significant changes in spicy compared to non-sound group 15 min after swimming $(P<0.05)$; (B) Hunger; *Significant change in spicy and light groups compared to before and ${ }^{* *}$ immediately after swimming $(\mathrm{P}<0.05)$; (C) Prospective; ${ }^{* *}$ Significant change in spicy and light groups compared to immediately after swimming; \#Significant changes in spicy compared to light music 15 min after swimming ( $<<0.05)$; (D) Satisfaction; *Significant change in spicy group compared to before swimming and $* *$ immediately after swimming; \#Significant difference in spicy compared to non-sound group $15 \mathrm{~min}$ and $1 \mathrm{~h}$ after swimming, and with light music group 15 and $30 \mathrm{~min}$ after swimming $(\mathrm{P}<0.05)$.

Therefore, the purpose of this study was to evaluate the effect of two types of spicy and light music in the recovery period.

As observed in previous studies the effect of music on lactate $[20,25]$, we hypothesized that a particular type of music (spicy and light) would have different effects on lactate clearance. Interestingly, in the minute 5 of the recovery, the music affected. That is, under fatigue and at the beginning of recovery, music did not have a beneficial effect, and it did not, at the 15 th minute.

This finding was not similar to Eliakim et al [20] research, as they observed the effect of the music at twelve and fifteen minutes, and stated that the positive effect of music was only evident in the recovery period as time elapsed. Because as time went by, fatigue and HR was reduced.

Probably the reason for the 15th minute inconsistency was due to the type of exercise. The exercise protocol of the present study was endurance, while their research exercise was intense, and endurance exercise than intense exercise does not affect too much lactate levels [26].

Because the exercise protocol of the present study is enduring in nature, it does not affect lactate levels more
[26], and for the same reason, at the 15th minute lactate was significantly reduced in all groups, even in the control group.

Physical exertion is caused by the duration and intensity of the exercise. In endurance exercise, fatigue occurs because of the long duration of the exercise. This fatigue has strong effects on mental states. Therefore, in the interplay of the psychological effects of music on the mind and fatigue on the mind, fatigue will overcome. So it was probably because of this factor that music didn't affect lactate after exercise [20].

Immediately after swimming, HR increased due to physical activity, and this increase is also noticeable in the minutes of 2, 5, 10 and 15 after swimming. However, in the second minute, there was a significant difference between the non-sound and the music groups. This indicated that the influence of music on the HR variations after a moderate time swimming. Probably in contrast between the mental effect of fatigue and the mental effect of music, the positive effects of music are temporarily dominant, the effect disappears in the fifth minute.

As time elapsed and fatigue decreased in the minutes of 10,15 and 25 after swimming, the fatigue decreased 
and the effects of music reappeared.

At minutes of 10, 15 and 25, there was a significant increase in spicy music compared to light music, which was probably due to the motivational state of spicy music that increased the HR [7].

Interestingly, over time, the effect of understanding music increased. And at twenty-five minutes after swimming the music significantly decreased the HR compared to the control group. Consistent with this finding, Savitha et al. concluded that light music after swimming is suitable for rest and helps to recover physical parameters and HR decrease [25].

In addition, there is a hypothesis that light music can also affect the HR by affecting the respiratory system [25]. So it is also likely that more effect of light music in the twenty-fifth minute (due to distance from exercise time) was likely to be due to breathing.

The results of this study showed that listening to music during the recovery period after swimming did not have a significant effect on BP. The SBP and DBP immediately after swimming, 2 and 5 (only DBP) minutes after swimming in three types of music was significantly higher than before swimming.

Savitha et al reported that listening to both spicy and light music during the recovery period after exercise, will significantly reduce the BP [23].

In our research, the lack of a significant effect of music on BP can be due to lactate and metabolites accumulation. On the other hand, there is also the possibility that music does not impressive affect BP due to the body's need to lower its temperature and increase the blood flow of the skin and active muscles.

So, in order to balance the hemostasis, it is necessary to maintain BP at a higher level than before swimming.

However, there was a significant difference in SBP between the two groups of spicy and light music. In the spicy music, BP was high because of the stress state of spicy music.
In the present study, appetite decreased after swimming in all three groups. But there was an increase from minutes 15 . That means independent of the music effect, exercise has an effect of appetite suppress. However, this suppression of appetite was temporary, and at other times of recovery and with increasing time interval from swimming, appetite increased. This finding is consistent with the findings of most previous studies [27-29]. In this respect, it is hypothesized that exercise is a negative energy balance. Exercise with this mechanism stimulates food intake behavior to rebuild depleted resources and restore energy balance [30,31]. In addition, the difference of fullness and satisfaction were meaningful in spicy music and an increase was observed in prospective statement compared with quiet music.

According to our results, the rhythm of music is important in appetite. Along with the results of the present study, we can point to Mc Elrea and Standing [32] research that increased the consumption of drinks with spicy music. This finding was also confirmed by other researchers [33-36].

\section{Conclusion}

In summary, listening to light music during recovery from endurance swimming was associated with decreased lactate levels and HR, but listening to spicy music increased HR and prospective. Also, music no impact on BP.

Whether these changes can useful the next training session or competition is still unclear and needs further investigation. And we suggest that, given the limitations of the present study, this study should be conducted over a longer period of time and following other exercises and by evaluating other relevant variables.

\section{Disclosure of interest}

The authors declare that they have no competing interest.

\section{References}

1. Hayakawa Y, Takada K, Miki H, Tanaka K. Effects of music on mood during bench stepping exercise. Perceptual and motor skills. 2000;90(1):307-14. https://doi.org/10.2466/pms.2000.90.1.307

2. Pates J, Karageorghis CI, Fryer R, Maynard I. Effects of asynchronous music on flow states and shooting performance among netball players. Psychology of Sport and Exercise. 2003;4(4):415-27. https://doi.org/10.1016/S1469-0292(02)00039-0

3. Corigliano B. What are the ergogenic effects of music during exercise?, Kinesiology, Sport Studies and Physical Education, Education Synthesis Projects. 2017; 26.

4. Tan F, Tengah A, Nee LY, Fredericks S. A study of the effect of relaxing music on heart rate recovery after exercise among healthy students. Complementary therapies in clinical practice. 2014;20(2):114-7. https://doi.org/10.1016/j.ctcp.2014.01.001

5. Edworthy J, Waring H. The effects of music tempo and loudness level on treadmill exercise. Ergonomics. 2006;49(15):1597-610. https://doi.org/10.1080/00140130600899104

6. Terry Peter C, Costas I Karageorghis. Music in sport and exercise. 2011.

7. Savitha D, Mallikarjuna RN, Rao C. Effect of different musical tempo on post-exercise recovery in young adults. Indian journal of physiology and pharmacology. 2010;54(1):32-6.

8. Masters KS, Ogles BM. Associative and dissociative cognitive strategies in exercise and running: 20 years later, what do we know? The sport psychologist. 1998;12(3):253-70. https://doi.org/10.1123/tsp.12.3.253

9. Crust L. Carry-over effects of music in an isometric muscular endurance task. Perceptual and motor skills. 2004;98(3):985-91. https://doi.org/10.2466/pms.98.3.985-991

10.Tenenbaum G. A social-cognitive perspective of perceived exertion and exertion tolerance. Handbook of sport psychology. 2001;2:810-822.

11.UrakawaK, YokoyamaK. Musiccan enhanceexercise-induced sympathetic dominancy assessed by heart rate variability. The Tohoku journal of experimental medicine. 2005;206(3):213-8. 
https://doi.org/10.1620/tjem.206.213

12.Tate AR, Gennings C, Hoffman RA, Strittmatter AP, Retchin SM. Effects of bone-conducted music on swimming performance. Journal of strength and conditioning research. 2012;26(4):982-988. https://doi.org/10.1519/JSC.0b013e31822dcdaf

13.Yarahmadi H, Haghighi A, Shojaei M, Beheshti Nasr S. Effect of nine weeks of moderate aerobic training on insulin resistance and appetite level in obese women. Quarterly of the Horizon of Medical Sciences. 2014;20(1):9-16.

14.Maraki M, Tsofliou F, Pitsiladis Y, Malkova D, Mutrie N, Higgins S. Acute effects of a single exercise class on appetite, energy intake and mood. Is there a time of day effect? Appetite. 2005;45(3):272-8. https://doi.org/10.1016/j.appet.2005.07.005

15.King JA, Miyashita M, Wasse LK, Stensel DJ. Influence of prolonged treadmill running on appetite, energy intake and circulating concentrations of acylated ghrelin. Appetite. 2010;54(3):492-8. https://doi.org/10.1016/j.appet.2010.02.002

16.Ghavam-Bakhtiar R, Nikbakht H, Ziaee N, Mohammadi $M$. The effect of relaxing music on changes in blood lactate level during recovery following a maximal exercise session in young female athletes. International Journal of Sports Medicine. 2012;2(9):432-5.

17.Jeffreys I.Amultidimensional approach to enhancing recovery. Strength and Conditioning Journal. 2005;27(5):78- 85. https://doi.org/10.1519/00126548-200510000-00014

18.Dodd S, Powers SK, Callender T, Brooks E. Blood lactate disappearance at various intensities of recovery exercise. Journal of Applied Physiology. 1984;57(5):1462-5. https://doi.org/10.1152/jappl.1984.57.5.1462

19.Hermansen L, Stensvold I. Production and removal of lactate during exercise in man. Acta Physiologica Scandinavica. 1972;86(2):191-201. https://doi.org/10.1111/j.1748-1716.1972.tb05325.x

20.Eliakim M, Bodner E, Eliakim A, Nemet D, Meckel Y. Effect of motivational music on lactate levels during recovery from intense exercise. The Journal of Strength \& Conditioning Research. 2012;26(1):80-6. https://doi.org/10.1519/JSC.0b013e31821d5f31

21.Lee D, Henderson A, Shum D. The effect of music on preprocedure anxiety in Hong Kong Chinese day patients. Journal of clinical Nursing. 2004;13(3):297-303. https://doi.org/10.1046/j.1365-2702.2003.00888.x

22.Szmedra L, Bacharach D. Effect of music on perceived exertion, plasma lactate, norepinephrine and cardiovascular hemodynamics during treadmill running. International journal of sports medicine. 1998;19(01):32-7. https://doi.org/10.1055/s-2007-971876

23.Boone T, Linderman JK, Astorino T, Baker J, Dalleck L, Drury D, et al. Cardiovascular responses to music tempo during steady-state exercise. Journal of Exercise Physiology Online. 2009; 12(1):50-56.

24.Pujol TJ, Langenfeld ME. Influence of music on Wingate Anaerobic Test performance.
Perceptual and motor skills. 1999;88(1):292-6. https://doi.org/10.2466/pms.1999.88.1.292

25.Bhavsar SD, Abhange RS, Afroz S. Effect of different musical tempo on post-exercise recovery in young adults. Journal of Dental and Medical Sciences. 2014;13(5):60- 4. https://doi.org/10.9790/0853-13516064

26.Ohkuwa T, Tsukamoto K, Yamai K, Itoh H, Yamazaki Y, Tsuda $T$. The relationship between exercise intensity and lactate concentration on the skin surface. International journal of biomedical science. 2009;5(1):23.-27.

27.Sim AY, Wallman K, Fairchild T, Guelfi K. High-intensity intermittent exercise attenuates ad-libitum energy intake. International journal of obesity. 2014;38(3):417-422. https://doi.org/10.1038/ijo.2013.102

28. Matos V, Souza D, Santos V, Medeiros Í, Browne R, Nascimento P, et al.. Acute effects of highintensity interval and moderate-intensity continuous exercise on glp-1, appetite and energy intake in obese men: A crossover trial. Nutrients. 2018;10(7):889. https://doi.org/10.3390/nu10070889

29.King N, Burley V, Blundell J. Exercise-induced suppression of appetite: effects on food intake and implications for energy balance. European journal of clinical nutrition. 1994;48(10):715-24.

30.Katsuki A, Sumida Y, Gabazza EC, Murashima S, Tanaka T, Furuta M, et al. Plasma levels of agouti-related protein are increased in obese men. The Journal of Clinical Endocrinology \& Metabolism. 2001;86(5):1921-4. https://doi.org/10.1210/jcem.86.5.7458

31. Shen CP, Wu K, Shearman L, Camacho R, Tota M, Fong $\mathrm{T}$, et al. Plasma agouti-related protein level: a possible correlation with fasted and fed states in humans and rats. Journal of neuroendocrinology. 2002;14(8):607-10. https://doi.org/10.1046/j.1365-2826.2002.00825.x

32.McElrea H, Standing L. Fast music causes fast drinking. Perceptual and Motor skills. 1992;75(2):362. https://doi.org/10.2466/PMS.75.5.362-362

33.Milliman RE. The influence of background music on the behavior of restaurant patrons. Journal of consumer research. 1986;13(2):286-9. https://doi.org/10.1086/209068

34.Caldwell C, Hibbert SA. The influence of music tempo and musical preference on restaurant patrons' behavior. Psychology \& Marketing. 2002;19(11):895-917. https://doi.org/10.1002/mar.10043

35.Wansink B, Van Ittersum K. Fast food restaurant lighting and music can reduce calorie intake and increase satisfaction. Psychological Reports. 2012;111(1):228-32. https://doi.org/10.2466/01.PR0.111.4.228-232

36.Novak CC, La Lopa J, Novak RE. Effects of sound pressure levels and sensitivity to noise on mood and behavioral intent in a controlled fine dining restaurant environment. Journal of Culinary Science \& Technology. 2010;8(4):191-218. https://doi.org/10.1080/15428052.2010.535756 


\section{Information about the authors:}

Javad Mehrabani; (Corresponding Author); https://orcid.org/0000-0001-7504-8066; mehrabanij@guilan.ac.ir; Department of Exercise Physiology, Faculty of Sport Sciences, University of Guilan ; Rasht, Guilan, Iran.

Soodabeh Bagherzadeh; https://orcid.org/0000-0001-9791-9782; soodabehbagherizadeh1@yahoo.com; Department of Physical Education and Sport Sciences, Faculty of Humanities, Islamic Azad University. ; Rasht, Guilan, Iran.

Abuzar Jorbonian; https://orcid.org/0000-0002-0447-1016; jorbonian_a@yahoo.com; Department of Exercise Physiology, Faculty of sport Sciences, University of Guilan ; Rasht, Guilan, Iran.

Eisa Khaleghi Mamaghani; https://orcid.org/0000-0002-0780-9211; khaleghimamaghani.eisa@yahoo.com; Department of Exercise Physiology, Faculty of sport Sciences, University of Guilan ; Rasht, Guilan, Iran.

Maryam Taghdiri; https://orcid.org/0000-0001-7161-2010; taghdiri.maryam.i@yahoo.com; Department of Exercise Physiology, Faculty of Sport Sciences, University of Mazandaran ; Babolsar, Mazandaran, Iran.

Mona Mehdizadeh Haghighi; https://orcid.org/0000-0003-1465-4497; mehdizadeh-haghighi@yahoo.com; Department of Physical Education and Sport Sciences, Faculty of Humanities, Islamic Azad University ; Rasht, Guilan, Iran.

Cite this article as:

Javad Mehrabani, Soodabeh Bagherzadeh, Aboozar Jorbonian, Eisa Khaleghi-Mamaghani, Maryam Taghdiri, Mona

Mehdizadeh-Haghighi. Cardiovascular, lactate and appetite response to light and spicy music tempo after an endurance swimming protocol in young girls. Pedagogy of physical culture and sports, 2020;24(4):195-202.

https://doi.org/10.15561/26649837.2020.0407

This is an Open Access article distributed under the terms of the Creative Commons Attribution License, which permits unrestricted use, distribution, and reproduction in any medium, provided the original work is properly cited (http://creativecommons.org/licenses/by/4.0/deed.en).

Received: 04.01.2020

Accepted: 25.02.2020; Published: 30.08 .2020 